\title{
Tri-plane correlation imaging for the detection of breast cancer: Effects of angular separation and correlation rule
}

\author{
Ehsan Samei ${ }^{1,2,3}$, Robert S. Saunders ${ }^{1}$, Swatee Singh $^{1}$, J oseph Y. Lo ${ }^{1,2}$, J ay A. Baker ${ }^{1}$ \\ 1. Carl E. Ravin Advanced Imaging Laboratories, Department of Radiology, Durham, NC, United States. 2. Department of \\ Biomedical Engineering, Department of Electrical and Computer Engineering, and Medical Physics Graduate Program, \\ Durham, NC, United States. 3. Department of Physics, Duke University, Durham, NC, United States.
}

Correspondence: Ehsan Samei. Address: Duke University, 2424 Erwin Road, Suite 302, Durham, NC 27705, United States. Email: samei@duke.edu.

Received: August 1, 2014

DOI : $10.5430 /$ ijdi.v2n2p29

Accepted: February 27, $2015 \quad$ Online Published: March 23, 2015

URL: http://dx.doi.org/10.5430/ijdi.v2n2p29

\section{Abstract}

Objective: Overlapping anatomy in projection mammography can degrade the detection of mammographic lesions and further create mass-like features that can confound computer-aided detection (CADe) systems and degrade their performance. Tri-plane correlation imaging (TCI) aims to reduce this impact by incorporating three projection images into CADe using a geometric correlation scheme. This study aimed to assess the efficacy of TCI for breast cancer detection.

Materials and Methods: An image set of 198 human subjects was used from a breast tomosynthesis database. Each case included 25 projections acquired within a 45 degree angular span at an approximate total glandular dose equal to that of two-view mammography. Triplet images were derived from each case (the central projection along with two symmetrical images) and analyzed by two independent CADe programs. The CADe results from the three projections from each program were combined using two TCI correlation rules based on unanimous vote and majority vote. The findings were analyzed in terms of true positives (TP), sensitivity, false positives per breast volume (FP), and a positive predictive index combining the two figures (i.e., $\mathrm{TP} /(\mathrm{TP}+\mathrm{FP})$ ).

Results: For the first CADe program, the TCI scheme using the majority voting rule improved sensitivity by $40 \%$ while maintaining specificity, leading to a $40 \%$ improvement in the PPI performance. For the second, a higher-sensitivity/ lower-specificity CADe program, the TCI using the majority voting rule improved sensitivity by $10 \%$ while increasing false positives per breast volume by $3 \%$, leading to an improvement of $8 \%$ in the PPI performance. The unanimous voting rule led to notably lower performance for both programs. For both CADe programs, an angular separation of six degrees ( \pm 3 degrees) proved optimal.

Conclusions: TCI was able to improve sensitivity over single projection imaging while maintaining specificity for both CADe programs, which suggests its potential as a supplement to standard mammography and as a complementary module to existing CADe algorithms.

\section{Keywords}

Mammography, Computer aided detection, Correlation imaging, Tomosynthesis 


\section{Introduction}

In standard mammography, overlapping anatomical structures can mask the presence of suspected lesions ${ }^{[1,2]}$. In certain conditions, overlapping anatomical structures can also mimic the appearance of breast masses in the formation of so called "noise lesions;" normal image features that appear like real lesions due to the confluence of unrelated anatomical structures ${ }^{[1,2]}$. These overlapping structures thus may confound mammographic computer-aided detection (CADe) systems leading to loss of sensitivity as well as specificity. To reduce these effects, recent technological developments have focused on removing anatomic overlap via breast CT and tomosynthesis ${ }^{[2-7]}$. While CT and tomosynthesis have significant potential, they may incur higher costs with complex equipment and image processing. Moreover, both CT and tomosynthesis devices produce multiple projection images and even more reconstructed slices. Such an increase in the number of images that a radiologist has to read affects radiologist workflow in a screening setting. An alternative low-cost and efficient technology is correlation imaging ${ }^{[8]}$. In correlation imaging, a standard projection image is geometrically correlated with additional projections to eliminate suspicious regions created from anatomical overlap, or otherwise to improve sensitivity at constant or improved specificity.

Correlation Imaging has been primarily applied to chest radiography ${ }^{[8-10]}$. A natural extension would be to explore the utility of the technique in breast imaging. However, while preliminary explored ${ }^{[11]}$, that has not been fully developed. Further, a key question in the implementation of correlation imaging is how the performance of the technique varies with respect to angular separation of the individual projections (i.e., the angular difference between the individual projections that comprise the correlation imaging technique). This paper reports on a study that sought to answer these questions, using low-dose human subject data, in the context of a tri-plane implementation of the technique for breast imaging, so-named Tri-plane Correlated Imaging (TCI). The study limited the number of projections used to three to facilitate a future implementation of the technology that might use stereoscopic viewing of the left-right projections ${ }^{[12]}$. Image data were derived from a database of low-dose tomosynthesis projections at five different angular separations. These projection images were each analyzed with two different CADe programs; the suspicious regions identified on each projection were then geometrically correlated. The performance results were then compared across different angular separations and with different correlation rules. A key objective of the study was to determine the extent by which the additional low-dose views could improve CADe performance compared to that on single-view data.

\section{Methods and materials}

\subsection{Human subject database}

This study was approved by the Institutional Review Board; informed consent was obtained for all subjects, and the study was compliant with the Health Insurance Portability and Accountability Act. An image database of 198 human subjects originally acquired for a clinical trial of tomosynthesis was used for this study ${ }^{[3]}$. The imaging system (MAMMOMAT Novation TOMO; Siemens Healthcare; Erlangen, Germany) used a selenium-based direct flat-panel detector with inherent $85 \mu \mathrm{m}$ pixels for image capture. Each case consisted of 25 projection images that were acquired over an angular range of 45 degrees (see Figure 1). To reduce computational complexity, raw image pixels were binned $4 \times 4$ to produce an effective pixel size of $340 \mu \mathrm{m}$ which was used for all image processing. All image sets were obtained using a W/Rh tube using 28-32 kVp.

From the 198 images, 145 were normal, and 53 had masses. Image data were acquired from the human subjects in both the mediolateral oblique (MLO) and the craniocaudal (CC) orientations (80 Left CC, 191 Left MLO, 81 Right CC, 186 Right MLO volumes). The breast densities and the subtly of the masses were representative of the population of patients at our institution. Specifically, the distribution of densities was $40 \%, 24 \%, 29 \%$, and $7 \%$, for the BI-RADS density categories of 1 to 4, respectively. Comparison standard (the "truth") was determined by an experienced MQSA radiologist (15 years' experience) who identified the lesions in all projection view images and determined the lesion extent. We did not establish 
a biopsy-based gold standard to ascertain the absolute presence of the lesions but our methodology was rigorous providing a reasonably robust alternative.

The projection images were obtained without any post-processing except for conversion to logarithmic scale to make image values linearized with respect to attenuation. All image analysis was done without processing applied for visual interpretation. However, to suppress the background and improve visualization at the breast skin line, each mammographic projection was processed using a simple gray scale transformation that has been previously verified to mimic clinical processing ${ }^{[13]}$. The mammogram gray scale histogram was then analyzed to identify the breast and background distributions. The histograms had identifiable features to separate the two distributions. These parameters were combined to create a sigmoid transform that was applied to each mammographic projection.

From the mammographic projections associated with each of 198 cases, the central projection image and projections at $\pm 3, \pm 6, \pm 12$, and \pm 22 were extracted to be incorporated in the TCI scheme, as illustrated in Figure 1. As each projection image was acquired at an exposure (and thus the glandular dose, considering a constant beam quality) approximately $8 \%$ of that of standard, single-view mammography, the total dose associated with the TCI exam, as modeled in this study, was about a quarter $(3 \times 8 \%=24 \%)$ of that of a single-view mammogram.

Figure 1. Diagram of TCI technique. Three images of the compressed breast are taken from three separate projects spanning an angular range of $\theta$ with respect to the center of rotation. The system used a source to image distance of $65.3 \mathrm{~cm}$, and an iso-centric gantry pivoting the x-ray tube about a point located $6 \mathrm{~cm}$ above the detector.

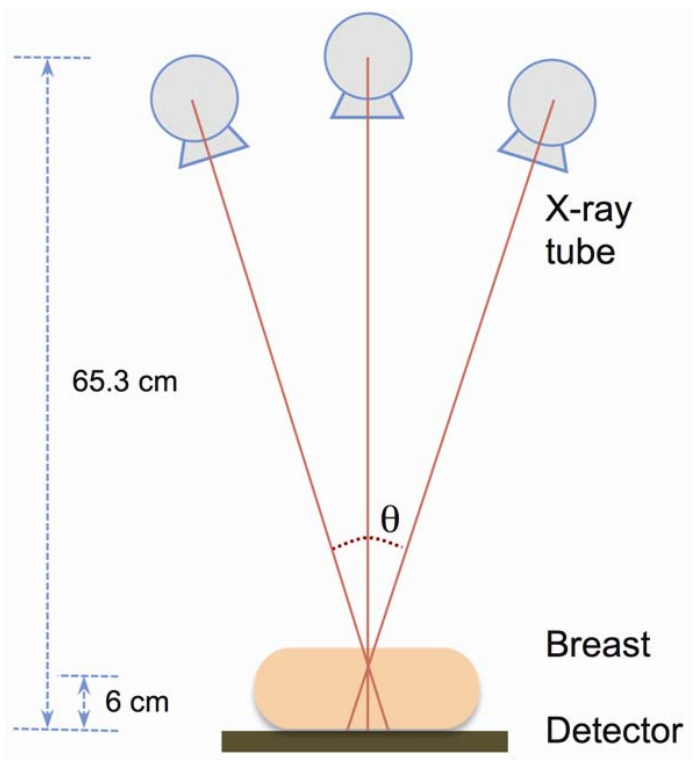

\subsection{I mage processing}

The tri-plane correlation process was studied using the mass detector outputs of two CADe algorithms. The use of two algorithms was motivated as a way to examine the robustness of the approach and the generalizability of the findings.

The first CADe system (CADe-1) filtered the images with a difference of Gaussians (DoG) filter ${ }^{[3]}$. This approach has been successfully used in CADe algorithms for mammography ${ }^{[14,15]}$. The DoG filter was formed by subtracting two Gaussians as

$$
w=\frac{1}{\sqrt{2 \pi \sigma_{S}^{2}}} e^{-r^{2} / 2 \sigma_{S}^{2}}-\frac{1}{\sqrt{2 \pi \sigma_{L}^{2}}} e^{-r^{2} / 2 \sigma_{L}^{2}},
$$


where $r$ corresponds to the radial distance from the origin, $\sigma_{L}$ represents the standard deviation of the larger Gaussian $(8.16 \mathrm{~mm})$, and $\sigma_{S}$ refers to the standard deviation of the smaller Gaussian $(4.76 \mathrm{~mm})$. This filtering enhanced (or boosted) the features of interest in the image. The filtered images were then segmented using adaptive thresholding to produce CADe suspicious locations. For each projection, the thresholds started with the top tenth percentile of the filtered pixel values and then lowered until any two suspicious locations were merged together. The joining rule was used as the stopping criterion of the thresholding process. The results of the overall TCI scheme was not sensitive to the adaptive thresholding process and stopping criteria, but as in any CAD approach, this front-end component produced a large number of false positives (FPs), suspicious image enhancements that did not correspond to "true" lesions. A rule-based false-positive approach, as implemented earlier ${ }^{[3]}$, was used to eliminate suspicious regions based on area, eccentricity, and filter values. This first mass detection algorithm had been previously optimized for our database with a reported sensitivity of $93 \%$ at 7.7 FPs per breast, corresponding to the algorithm results for the entire breast volume ${ }^{[3]}$.

The second CADe system (CADe-2) was implemented as described in an earlier publication ${ }^{[11]}$. In summary, the system filtered the projection images with a modified adaptive elliptical gradient convergence filter, based on the convergence index filter approach, to highlight suspicious regions. In the segmentation step, the filtered image data were smoothed using a Gaussian filter $(\sigma=3.5 \mathrm{~mm})$ to minimize the enhancements that were associated with (comparatively smaller-sized) ducts, while retaining the areas of enhancement that would likely be associated with (comparatively larger-sized) masses ${ }^{[11]}$. Suspicious regions in the filtered image were segmented using a gray scale duration process. The filtered image was first thresholded at the 99th percentile. The threshold continued to be lowered to each lower percentile to grow regions until the entire region was segmented or a region attempted to merge with another suspicious region ${ }^{[16]}$. A minimal rule-based false-positive approach was used to eliminate suspicious regions based on area and eccentricity.

The TCI algorithm combined the information from each set of three breast projections associated with each case. Figure 2 illustrates the major steps in the TCI geometric correlation algorithm. Each projection contained suspicious regions identified by each of the CADe systems. These suspicious regions were first binarized to unity value at lesion location and zero elsewhere. The binarized images were then back-projected into the breast volume based on the imaging system geometry using the shift-and-add technique ${ }^{[3,11]}$. The breast volume is discretized with $2 \mathrm{~mm}$ slice thickness, a magnitude found to adequately reflect the suspicious lesions of the targeted size. The result of this reconstruction was a volume containing voxel values corresponding to $0,1,2$, or 3 depending on how many projections contributed toward each voxel. The back-projected volume was then thresholded to include only voxels shared by all projections (voxel values $=3$ ), applying a "unanimous voting" rule. The data also thresholded to include only voxels shared by majority of projections (voxel values $=2-3$ ), applying a so-called "majority voting" rule. The thresholded volumes were then summed in the $\mathrm{z}$ direction, equivalent to a parallel beam approximation of the central projection angle, to produce a single TCI projection.

Figure 2. Overview of TCI geometric correlation algorithm. First $\mathrm{CAD}$ is applied to individual projections (left). The individual CAD results are the combined by reconstruction (center). Finally the combined data is projected into the planar domain to identify the overall output of the processing for the case (right).

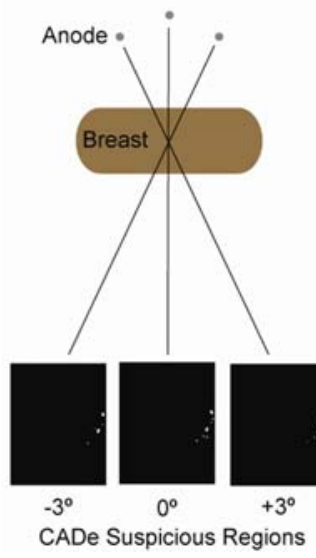

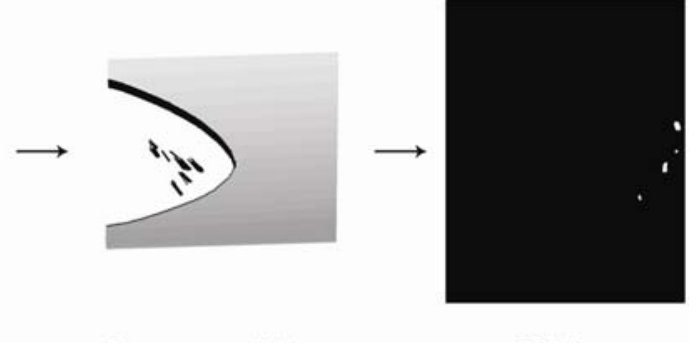

Reconstructed Volume
TCl Output 


\subsection{Performance evaluation}

The performance of the CADe programs on single-view projections and the TCI results were assessed in terms of sensitivity and specificity. For the evaluations, the conformance with the comparison standard (the "truth") was determined based on percentage overlap of the identified suspicious regions with the "truth." If a suspicious region overlapped a mass by more than or equal to $30 \%$, it was considered a hit:

$$
\frac{S_{\text {Area }} \cap T_{\text {Area }}}{S_{\text {Area }}} \geq 30 \%
$$

where $\mathrm{S}_{\text {Area }}$ represents the area of a suspicious region identified by a CADe algorithm and $\mathrm{T}_{\text {Area }}$ describes the true area of a mass delineated by an experienced radiologist. This rule was chosen as it ensured that the suspicious region was focusing on the abnormality while penalizing suspicious regions that blanketed large portions of the image. The $30 \%$ rule was empirically found to accommodate a possible shift in the center location of a legion depending on how the lesion might coincide with the underlying anatomical structure. The registration of the TCI output and the truth neglected the slight difference in the projection geometries (cone beam versus parallel bream), as that was an order of magnitude lower than the $30 \%$ rule that was applied.

The sensitivity and FP per breast volume were combined into one figure of merit, the positive predictive index (PPI) ${ }^{[8,9]}$. This was defined as

$$
P P I=\frac{\text { sensitivity } \cdot p}{\text { sensitivity } \cdot p+\frac{F P s}{\text { Volume }}}
$$

where $p$ represents the breast cancer prevalence in a screening population. The prevalence was chosen to be $0.78 \%$ based on previously published studies ${ }^{[17]}$. The PPI describes how likely a suspicious region identified by a CADe algorithm would be a cancer in a screening population.

The CADe baseline performance was defined for the system operating only on the central $\left(0^{\circ}\right)$ projection. System performance was then measured for the correlated images produced at multiple angular separations and TCI voting rules. The sensitivity, FP/breast volume, and PPI were converted into relative values to measure the utility of the TCI algorithm in relation to the baseline single-view, CADe performance.

\section{Results}

Figure 3 illustrates the effects of angular separation and voting rule on sensitivity, false positives per breast volume, and positive predictive index for the CADe- 1 system ${ }^{[1]}$. For a unanimous voting rule, increased angular separation reduced the number of FPs per breast volume as the chance correlation between randomly matched features reduced. However, that was at the expense of sensitivity; increased angular separation with a unanimous voting rule led to a lower positive predictive index. For a majority voting rule, increased angular separation increased sensitivity at the expense of increased FPs per breast volume. Overall, the positive predictive value increased to a maximum at \pm 3 degrees angular separation but declined beyond that. The optimality of \pm 3 degrees angular separation coincides with prior reports ${ }^{[8,11]}$, and incidentally is in the range applicable for stereoscopic visualization ${ }^{[12]}$.

Figure 4 illustrates the effects of angular separation and voting rule on sensitivity, FPs per breast volume, and positive predictive index for the CADe-2 system. For a unanimous voting rule, increased angular separation slightly reduced the number of false positives per breast volume while moderately lowering sensitivity. The PPI increased to a maximum at \pm 3 degrees and then declined. For a majority voting rule, sensitivity increased with angular separation till reaching a 
maximum at \pm 6 degrees, beyond which it declined. This sensitivity benefit was achieved with a minor increase in FPs per breast volume. Overall, similar to the finding with the other CADe algorithm, the positive predictive value increased to a maximum at \pm 3 degrees angular separation but declined beyond that.

For comparative purposes, the CADe-1 program, when applied only to the zero-degree low-dose projections, it provided a sensitivity of $60.0 \%$ at $6.3 \mathrm{FPs} /$ image. The corresponding figures for the CADe- 2 program were $53.0 \%$ sensitivity at 8.0 FPs/image. These are intermediate results, as these CADe programs were not designed for use at $8 \%$ of single-view mammographic dose (even if the noise is reduced by pixel binning). Even though the CADe-1 program was previously planned for such data, it was designed to be applied to the entire projection dataset, not to a sub-selection of the projections. As such, these performance metrics cannot be directly compared with those from CADe systems designed to operate on full dose mammograms. However, they establish the baseline performance that TCI sought to improve. Furthermore, the comparison with the single-view, zero-degree projection views (with an acquisition dose lower than that of the combined TCI system), was made as a point of reference only, further justified by an underlying assumption that the main factor limiting mass detection in projection breast images is anatomical overlap, largely not impacted by the associated dose settings.

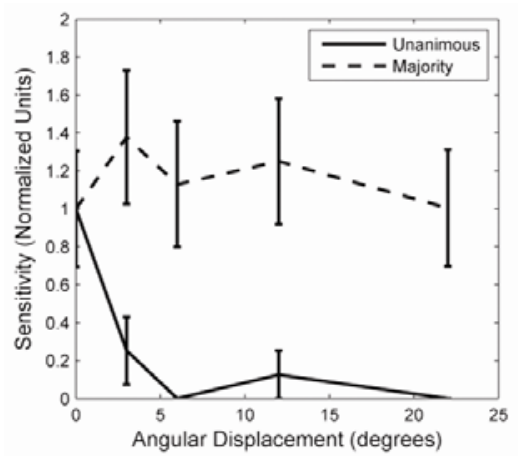

(a)

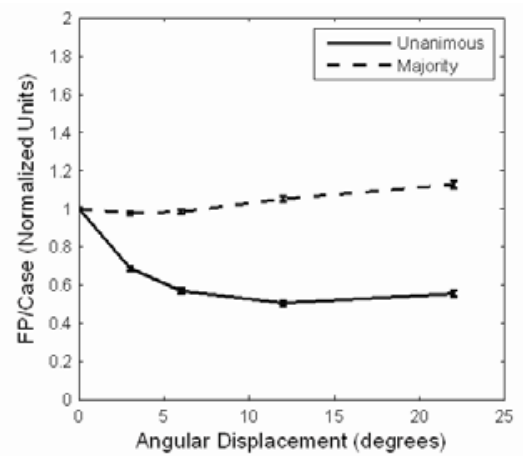

(b)

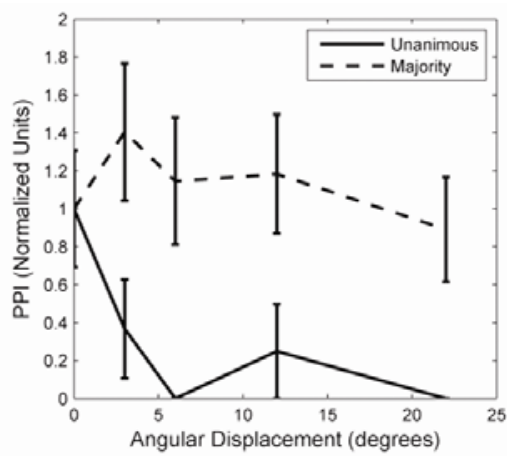

(c)

Figure 3. Effect of TCI algorithm on CADe sensitivity (a), non-existent false positives (FPs) per breast volume (b), and positive predictive index (c) for an optimized CADe system (CADe-1). The angular displacement is reflective of the angular separation of each of the oblique, left or right views from the central view. The data are normalized to unity for the single projection view, and error bars reflect the statistical uncertainty across the ensemble of cases used.

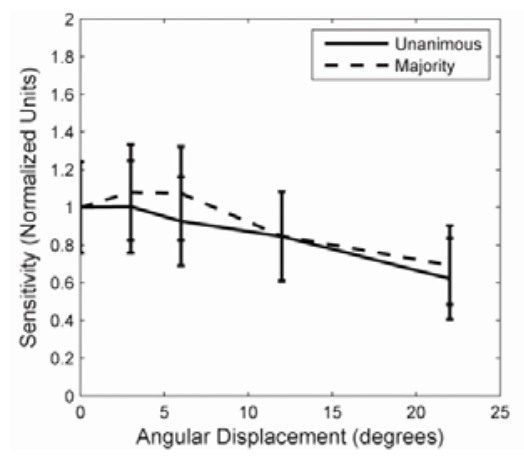

(a)

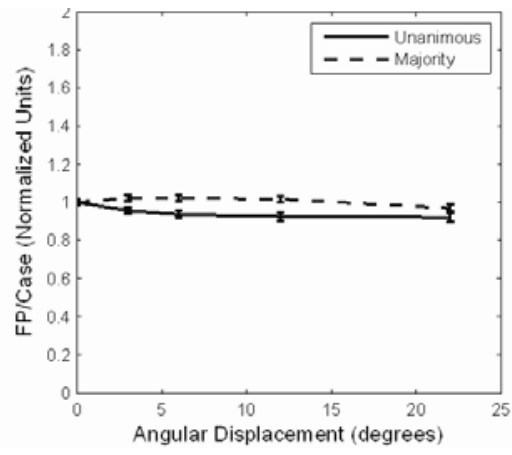

(b)

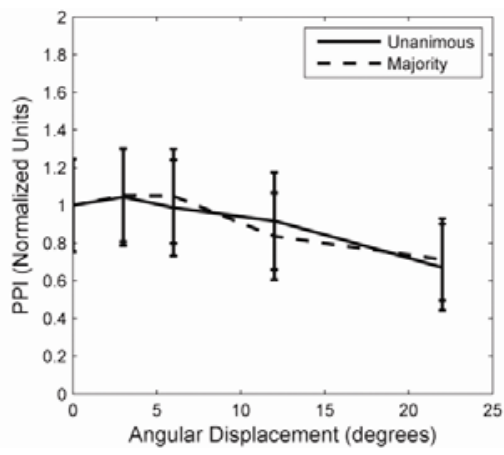

(c)

Figure 4. Effect of TCI algorithm on CADe sensitivity (a), non-existent false positives (FPs) per breast volume (b), and positive predictive index (c) for a basic CADe system (CADe-2). The angular displacement is reflective of the angular separation of each of the oblique, left or right views from the central view. The data are normalized to unity for the single projection view, and error bars reflect the statistical uncertainty across the ensemble of cases used. 


\section{Discussion}

Breast imaging using x-ray based methods remain an active area of research highlighted by recent studies that discuss its effectiveness and explore adjunct technologies that can improve its utility and potential ${ }^{[18-20]}$. Breast tomosynthesis has been a significant development in that front with the first commercial system recently approved by the FDA for clinical use in the United States. Investigators have further aimed to apply CAD methods to tomosynthesis datasets. Among them there has been adaptation of mammography algorithms to work with tomosynthesis ${ }^{[21]}$, combination of projection images with $3 \mathrm{D}$ volumes ${ }^{[22]}$, and a host of other implementations ${ }^{[23-26]}$. This study investigated the feasibility of a new adjunct technique to mammography: Tri-plane Correlation Imaging of the breast.

For a CADe system that was previously optimized for complete view breast tomosynthesis imaging (CADe-1) ${ }^{[1]}$, TCI using a majority voting rule improved sensitivity by $40 \%$ while maintaining specificity, leading to a $40 \%$ improvement in PPI performance. For the second CADe program (CADe-2), TCI using a majority voting rule improved sensitivity by $10 \%$ while increasing FPs per breast volume by $3 \%$, leading to an improvement of $8 \%$ in PPI performance. For both CADe programs, the optimal TCI set was the central projection paired with the projections acquired at \pm 3 degrees. TCI was able to improve sensitivity over single projection imaging while maintaining specificity, for two different CADe systems, which were not explicitly optimized for the tested dose levels. The findings indicate the potential of TCI as a beneficial supplement to standard mammography and a complementary module to existing CADe algorithms. Further improvement may be expected with acquisition and processing optimized for TCI including the acquisition of higher dose projection data. While these results are promising, it should be pointed out that CAD prompts, as advantageous as they might be, can lead to perceptual or cognitive responses that can reduce those advantages. Studies have noted that even a superior CAD might be sub optimally utilized in the clinical practice. These perceptual and cognitive elements of CAD require distinct and dedicated research of their own. The scope of the present study was limited to only a demonstration of the potential utility of the technology and not its clinical implementation.

Although the implementation of TCI in this study is new, it corresponds closely with the work of Reiser et al. ${ }^{[3]}$ where a multi-plane correlation across a wide range of angular views demonstrated the tradeoff between sensitivity and specificity for two correlation methods. A realization of the method was also previously implemented in chest radiography ${ }^{[8,9]}$. Prior studies in chest and breast radiography ${ }^{[8,9,12]}$, using human and phantom images, found that using the central projection along with projections acquired at $\pm 3-6$ degrees produced the best performance. The current study similarly found that an angular separation in the $\pm 3-6$ degrees yields optimal results. The current study added to prior work and also examined different voting rules (unanimous and majority) to determine whether regions were geometrically correlated. Other correlation imaging studies have examined the angular range and number of projections to combine ${ }^{[27,28]}$. Those studies have found that optimum performance tends to occur for 11-17 projections over an angular span of $45^{\circ}$. However, this work shows that performance improvements can be seen with as few as three projection images over a smaller angular arc.

Anatomical noise is a major limitation of projection imaging. Our study showed that the ensemble of three projections can offer an effective improvement over a single view to mitigate the anatomical noise in each view. Each of the three views renders the anatomical noise differently, and thus the combination of the three reduces the effect. Additional benefit can be gained by more projections with improvement patterns similar to those with three projection, but with diminishing return with each additional views, as shown in earlier publications ${ }^{[27,28]}$. Added projection implies reduced quantum noise within the ensemble of images, but as the anatomical noise is the main factor limiting mass detection in projection breast images ${ }^{[1,2]}$, the added advantage is significantly more than what would be expected from quantum noise differences. While the study ascertains this added advantage of using multiple views, it does not explicitly indicate how the technology can be used. As far as clinical implementation, we do not envision that a radiologist would necessarily look at the three views (unless a stereo display is incorporated); the complementary projection information is rather integrated by the computer algorithm to provide an improved CADe prompt to the observer. And the technique may be applied to any view mammogram, ie, each of the two view mammograms can individually processed the same way. 
In this study, the tri-plane geometric correlation algorithm was tested for two CADe algorithms. As both programs operate on very low-dose mammographic images extracted from a tomosynthesis database, their inherent performance on single images cannot be readily compared to those of commercial CADe algorithms designed for full dose mammograms. However, for both programs, false positives decreased with a unanimous rule and slightly increased with a majority correlation rule. For the first program (CADe-1), sensitivity dropped sharply with the unanimous rule but improved with a majority rule. This suggests that in cases where the CADe algorithm detected a mass, it detected it on most projections but not all projections. This is consistent with its more aggressive false-positive reduction algorithm. In contrast, the second CADe program (CADe-2) had a smaller difference in sensitivity between the two correlation rules. Sensitivity still improved with the majority rule and decreased slightly with the unanimous rule. This is consistent with its less aggressive false-positive reduction stage. In addition, CADe-1 demonstrated more fluctuation in performance as a function of angular separation, including a dip in performance at \pm 6 degree, compared to CADe-2. The fact that the different CADe's showed different performance patterns indicates that this was not an issue with the images. The fluctuations were also well within the error bars noted so it should not be taken as a systematic aberration. Future studies should consider more images and additional CADe programs to more fully substantiate the robustness of the technique. Nevertheless, the overall similarity of the results across CADe programs demonstrates the relative independence of the $\mathrm{CI}$ approach from the starting CADe algorithm used, demonstrating its general robustness.

In our study, each projection image was acquired at a low dose level ( $8 \%$ of standard single-view mammography), and as such the level of quantum noise in each projection was high. While anatomical noise is the main factor limiting the detection of mammographic masses, further implied by this study, quantum noise can still degrade CADe performance. An earlier study ${ }^{[27]}$ shows a 0.1 reduction in Az with $8 \%$ reduction is dose. Acquiring the projections at higher doses (ideally one-third of mammography dose) would improve the projection results. While this was not a scenario explored in this investigation, we hypothesize that reduced quantum noise can reduce the number of suspicious regions in the single-view projections and thus reduce the likelihood of coincidence match to cause false positives in TCI. This remains the topic of a follow up investigation. Furthermore, regardless of the level of dose, care should be exercised for the CADe algorithm to be explicitly trained for the dose level designed to be used with the imaging system; a CADe designed for full dose mammography might not be optimum for TCI or breast tomosynthesis.

This study faced several limitations. First, the current implementation of correlation imaging was limited to three projections to facilitate future stereoscopic viewing of the projection data ${ }^{[12]}$. However, correlation imaging can be implemented with more than three projections ${ }^{[27,28]}$ in order to improve performance and reduce CADe reconstruction artifacts. Second, the two CAD algorithms used showed differences some of which exhibited fluctuations that were random in nature. The study will benefit with additional CAD implementations, further stratifying the impact of breast volume on performance as well. Nonetheless, certain conclusions can be reasonably drawn from the results, in spite of their limitations in terms of the fluctuations and the number of algorithms tests, in that 1) a level of benefit can be gained by combining CAD results from multiple projections regardless of the CAD algorithm used, and that 2) the optimum angular separation tends to be in the 3-6 degree zone. Finally, future work should incorporate a larger number of human subject cases, in order to allow for a more complete statistical evaluation of the technique and stratification in terms of different breast tissue types. In that vain, a clinical study is also needed to compare the technique to two-view mammography or breast tomosynthesis in order to establish the quantitative merits of the technique compared to the standard of practice. Nevertheless, this study showed the potential of the TCI technique in improving CADe performance. The effectiveness of TCI is dependent on the CAD algorithm applied as well as the magnitude of angular separation. However TCI leads to a net improvement in performance at \pm 3 degrees angular separation.

\section{Conclusion}

In conclusion, this study found that with an optimized choice of angular separation and a correlation voting rule, TCI was able to improve sensitivity over the single projection approach while maintaining specificity (indicated by a net gain in PPI when TCI is invoked). The optimal angular separation was six degrees ( \pm 3 degrees $)$ and the optimal voting rule was a 
majority vote. By adding only two additional projections to a standard mammographic view, the positive predictive index could be improved by at least $8 \%$, suggesting that the TCI technique is a cost-effective means of improving mammographic performance. As the optimal angular range of 6 degrees is also optimal for stereoscopic viewing of the image pairs ${ }^{[12]}$, the TCI data could be easily utilized for stereoscopic mammography applications as well.

\section{Acknowledgements}

The authors wish to thank Brian Harrawood for computer support. This work was supported in part by grants from the Komen Foundation (PDF55806), NIH (R01-CA-109074 and R01-CA-112437), the Prevent Cancer Foundation, and a research agreement with Siemens Healthcare.

\section{References}

[1] Burgess AE, Jacobson FL, Judy PF. Human observer detection experiments with mammograms and power-law noise. Med Phys. 2001; 28(4): 419-37. PMid:11339738 http://dx.doi.org/10.1118/1.1355308

[2] Kiarashi N, Samei E. Digital breast tomosynthesis: a concise overview. Imaging Med. 2013; 5: 467-76. http://dx.doi.org/10.2217/iim.13.52

[3] Singh S, Tourassi GD, Baker JA, et al. Automated breast mass detection in 3D reconstructed tomosynthesis volumes: A featureless approach. Med Phys. 2008; 35: 3626-3636. PMid:18777923 http://dx.doi.org/10.1118/1.2953562

[4] Rafferty EA, Park JM, Philpotts LE, et al. Assessing Radiologist Performance Using Combined Digital Mammography and Breast Tomosynthesis Compared with Digital Mammography Alone: Results of a Multicenter, Multireader Trial. Radiology. 2013; 266: 104-113. PMid:23169790 http://dx.doi.org/10.1148/radiol.12120674

[5] Reiser I, Nishikawa RM, Giger ML, et al. Computerized mass detection for digital breast tomosynthesis directly from the projection images. Med Phys. 2006; 33: 482-91. PMid:16532956 http://dx.doi.org/10.1118/1.2163390

[6] Skaane P, Bandos AI, Eben EB, et al. Two-View Digital Breast Tomosynthesis Screening with Synthetically Reconstructed Projection Images: Comparison with Digital Breast Tomosynthesis with Full-Field Digital Mammographic Images. Radiology. 2014; 271: 665-3. PMid:24484063 http://dx.doi.org/10.1148/radiol.13131391

[7] Lindfors KK, Boone JM, Nelson TR, et al. Dedicated breast CT: initial clinical experience. Radiology. 2008; $246(3)$ : 725-33. PMid:18195383 http://dx.doi.org/10.1148/radiol.2463070410

[8] Samei E, Catarious JDM, Baydush AH, et al. Bi-plane correlation imaging for improved detection of lung nodules. Proc. SPIE. 2003; 5030: 284. http://dx.doi.org/10.1117/12.480478

[9] Nasab NM, Samei E. The impact of angular separation on the performance of biplane correlation imaging for lung nodule detection. Proc. SPIE. 2006; 6142: 61421A. http://dx.doi.org/10.1117/12.652588

[10] Samei E, Stebbins SA, Dobbins JT, 3rd, Lo JY. Multiprojection correlation imaging for improved detection of pulmonary nodules. AJR. 2007; 188: 1239-45. PMid:17449766 http://dx.doi.org/10.2214/AJR.06.0843

[11] Saunders RS, Jr, Samei E, Majdi-Nasab N, et al. Initial human subject results for breast bi-plane correlation imaging technique. Proc. SPIE. 2007; 6514: 651423-651427. http://dx.doi.org/10.1117/12.713722

[12] Webb L, Samei E, Lo JY, et al. Comparative performance of multi-view stereoscopic and mammographic display modalities for breast lesion detection. Medical Physics. 2011; 38(4): 1972-80. PMid:21626930 http://dx.doi.org/10.1118/1.3562901

[13] Saunders RS, Samei E, Baker J, et al. Comparison of LCD and CRT displays based on efficacy for digital mammography. Acad Radiol. 2006; 13: 1317. PMid:17070449 http://dx.doi.org/10.1016/j.acra.2006.07.017

[14] Catarious JDM, Baydush AH, Floyd JCE. Characterization of difference of Gaussian filters in the detection of mammographic regions. Med Phys. 2006; 33: 4104. PMid:17153390 http://dx.doi.org/10.1118/1.2358326

[15] Oliver A, Freixenet J, Martí J, et al. A review of automatic mass detection and segmentation in mammographic images. Med Image Anal. 2010; 14: 87-110. PMid:20071209 http://dx.doi.org/10.1016/j.media.2009.12.005

[16] Catarious JDM, Baydush AH, Floyd JCE. Incorporation of an iterative, linear segmentation routine into a mammographic mass CAD system. Med Phys. 2004; 31: 1512-20. PMid:15259655 http://dx.doi.org/10.1118/1.1738960

[17] Pisano ED, Gatsonis C, Hendrick E, et al. Diagnostic performance of digital versus film mammography for breast-cancer screening. N Engl J Med. 2005; 353: 1773-1783. PMid:16169887 http://dx.doi.org/10.1056/NEJMoa052911

[18] Nees AV. Digital mammography: are there advantages in screening for breast cancer? Acad Radiol. 2008; 15: $401-7$. PMid:18342763 http://dx.doi.org/10.1016/j.acra.2008.01.004 
[19] Zuley ML, Bandos AI, Abrams GS, et al. Time to diagnosis and performance levels during repeat interpretations of digital breast tomosynthesis: preliminary observations. Acad Radiol. 2010; 17: 450-5. PMid:20036584 http://dx.doi.org/10.1016/j.acra.2009.11.011

[20] Kontos D, Bakic PR, Carton AK, et al. Parenchymal texture analysis in digital breast tomosynthesis for breast cancer risk estimation: a preliminary study. Acad Radiol. 2009; 16: 283-98. PMid:19201357 http://dx.doi.org/10.1016/j.acra.2008.08.014

[21] Reiser I, Nishikawa RM, Giger ML, et al. Computerized mass detection for digital breast tomosynthesis directly from the projection images. Med Phys. 2006; 33: 482-91. PMid:16532956 http://dx.doi.org/10.1118/1.2163390

[22] Chan H-P, Wei J, Zhang Y, et al. Computer-aided detection of masses in digital tomosynthesis mammography: combination of 3D and 2D detection information. Proc. SPIE. 2007; 6514: 651416-651416. http://dx.doi.org/10.1117/12.713640

[23] Chan H-P, Wei J, Sahiner B, et al. Computer-aided detection system for breast masses on digital tomosynthesis mammograms: preliminary experience. Radiology. 2005; 237: 1075-80. PMid:16237141 http://dx.doi.org/10.1148/radiol.2373041657

[24] Bernard S, Muller S, Peters G, et al. Fast microcalcification detection on digital breast tomosynthesis datasets. Proc. SPIE. 2007; 6514: 65141X-65148. http://dx.doi.org/10.1117/12.709987

[25] Peters G, Muller S, Grosjean B, et al. A hybrid active contour model for mass detection in digital breast tomosynthesis. Proc. SPIE. 2007; 6514: 65141V-65111. http://dx.doi.org/10.1117/12.709593

[26] Wheeler FW, Perera AGA, Claus BE, et al. Micro-calcification detection in digital tomosynthesis mammography. Proc. SPIE. 2006; 6144: 614420-614412. http://dx.doi.org/10.1117/12.653478

[27] Chawla AS, Samei E, Saunders RS, et al. A mathematical model platform for optimizing a multiprojection breast imaging system. Med Phys. 2008; 35: 1337-1345. PMid:18491528 http://dx.doi.org/10.1118/1.2885367

[28] Chawla AS, Saunders RS, Singh S, et al. Towards optimized acquisition scheme for multiprojection correlation imaging of breast cancer. Acad Radiol. 2009; 16: 456-463. PMid:19268858 http://dx.doi.org/10.1016/j.acra.2008.09.013 